\title{
Cutinase A of Botrytis cinerea is Expressed, but not Essential, During Penetration of Gerbera and Tomato
}

\author{
J. A. L. van Kan, J. W. van't Klooster, C. A. M. Wagemakers, D. C. T. Dees, C. J. B. van der Vlugt- \\ Bergmans \\ Department of Phytopathology, Wageningen Agricultural University, P.O. Box 8025, 6700 EE Wageningen, \\ The Netherlands \\ Received 20 May 1996. Accepted 30 October 1996.
}

The plant pathogen Botrytis cinerea can infect undamaged plant tissue directly by penetration of the cuticle. This penetration has been suggested to be enzyme-mediated, and an important role for cutinase in the infection process has been proposed. In this study the expression of the cutinase encoding gene cutA of $B$. cinerea was analyzed using a cutA promoter-GUS reporter gene fusion. Transformants containing the fusion construct were examined for GUS expression on gerbera flowers and tomato fruits. High GUS activity was detected from the onset of conidial germination and during penetration into epidermal cells, indicating that cutA is expressed during the early stages of infection. To determine the biological relevance of cutinase A for successful penetration, cutinase A-deficient mutants were constructed by gene disruption. Pathogenicity of two transformants lacking a functional cutA gene was studied on gerbera flowers and tomato fruits. Their ability to penetrate and cause symptoms was unaltered compared to the wild-type strain. These results exclude an important role for cutinase A during direct penetration of host tissue by $B$. cinerea.

Additional keywords: gene disruption, grey mold, homologous recombination, pathogenicity gene, reporter gene.

The ubiquitous plant pathogen Botrytis cinerea Pers.:Fr. infects fruits, flowers, or green tissue of more than 200 plant species (Jarvis 1977). Direct penetration by germ tubes into undamaged plant tissue occurs via natural openings or through the cuticle (Verhoeff 1980). To breach the cutin layer, mechanical or enzymatic mechanisms have been suggested. Clark and Lorbeer (1976) reported the formation of appressorium-like structures that mediated penetration of onion leaves by mechanical force. McKeen (1974) and Rijkenberg et al. (1980) studied penetration of the cuticle of bean leaves and tomato fruits by electron microscopy. They observed a sharp pore without curled edges and no indentation of the cuticle or

Corresponding author: J.A.L. van Kan;

E-mail: jan.vankan@medew.fyto.wau.nl

Present address of C. J. B. van der Vlugt-Bergmans: Wageningen Agricultural University, Department of Food Science, Division of Industrial Microbiology , P.O. Box 8129, 6700 EV Wageningen, The Netherlands.

Accession number Z69263 of the GenEMBL database. epidermal wall during penetration by the infection peg. Moreover, the absence of appressorium-like structures indicated that penetration is an enzymatic process. Esterase activity at the tip of germ tubes (McKeen 1974) and the production of cutinase in liquid culture (Shishiyama et al. 1970; Baker and Bateman 1978) is consistent with a role for cutinase in penetration of the cuticle by $B$. cinerea. Involvement of cutinase in penetration by $B$. cinerea was indicated by the observation that monoclonal antibodies, raised against cutinase, reduced lesion formation on gerbera flowers by $80 \%$ when flowers were treated with this antibody after inoculation with conidia (Salinas 1992).

The cloning of the cutinase-encoding gene cutA of B. cinerea (Van der Vlugt-Bergmans et al. 1997) enables the use of a promoter-reporter gene fusion to investigate $c u t A$ gene expression during penetration of host tissue. The uidA reporter gene encoding $\beta$-glucuronidase (GUS, Jefferson et al. 1987) has been successfully used to study gene expression in fungal pathogens during colonization of host tissue (van den Ackerveken et al. 1994; Wubben et al. 1994). In this study, the GUS reporter system was employed to determine whether cutinase $\mathrm{A}$ is indeed produced by $B$. cinerea during penetration of host tissue, visualized as blue staining of fungal structures when GUS is expressed under control of the cutA promoter. To unequivocally determine the biological relevance of cutinase A for B. cinerea during penetration of its hosts, cutinase A-deficient mutants are required. The establishment of a transformation system for B. cinerea (Hilber et al. 1994; Hamada et al. 1994) and the availability of haploid $B$. cinerea strains (Büttner et al. 1994) allowed disruption of the single copy gene cutA, to obtain such mutants. Transformants lacking a functional $c u t A$ gene were evaluated for their ability to infect gerbera and tomato tissue.

\section{RESULTS}

Characterization of reporter gene transformants.

A fusion was made between the cutA promoter and the uidA reporter gene encoding $\beta$-glucuronidase (GUS, Jefferson et al. 1987). The terminator sequence of the tubA gene of $B$. cinerea (TtubA) was cloned upstream of the $c u t A$ promoter (PcutA) to minimize interference by regulatory sequences near the site of integration (Fig. 1A). The trpC terminator sequence from $A s-$ pergillus nidulans (TtrpC) was placed downstream of uidA. The resulting construct (pCutGUS) was introduced into $B$. 
cinerea strain SAS56 by cotransformation with the plasmid pOHT. Twelve hygromycin B resistant transformants were analyzed for the presence of pCutGUS insertions by PCR using primers complementary to the tubA terminator sequence and the uidA coding sequence (Fig. 1A). In 10 of the 12 transformants a fragment of $2.0 \mathrm{~kb}$ was amplified, indicating that the cutA promoter-uidA reporter gene fusion was correctly cotransformed into the genome of $B$. cinerea. The number of pCutGUS insertions was determined by Southern blot analysis (Fig. 2). A blot containing genomic DNA of five transformants (T1, T2, T3, T5, T7) and recipient strain SAS56 digested with EcoRV was probed with an EcoRV/HindIII fragment of pCutGUS (Fig. 1A) containing parts of the uidA coding region and $\operatorname{trp} \mathrm{C}$ terminator. $\mathrm{T} 2$ and $\mathrm{T} 5$ contained a single hybridizing fragment whereas $\mathrm{T} 1, \mathrm{~T} 3$, and $\mathrm{T} 7$ contained a large number of hybridizing fragments.

To assess whether these transformants exhibit GUS activity upon induction with the cutin monomer 16-hha (van der Vlugt-Bergmans et al. 1997), transformants and the wild-type strain SAS56 were grown in liquid B5 medium containing $0.3 \%$ glucose. After 2 days of growth 16-hha was added (day 0 ) and incubation continued for 3 days. Samples were taken for intracellular GUS activity measurements. Low GUS activ-
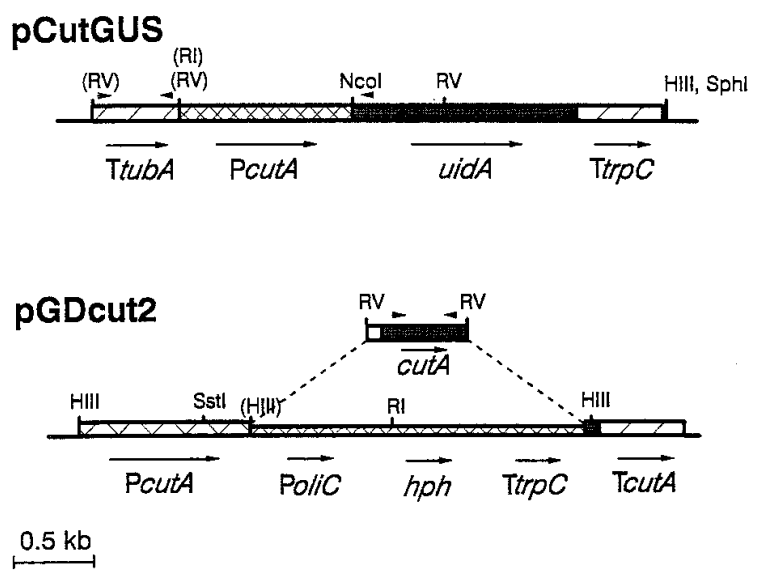

Fig. 1. Schematic representation of plasmids pCutGUS and pGDcut2 used for transformation of Botrytis cinerea. TtubA represents a terminator sequence of the $B$. cinerea $\beta$-tubulin gene. PcutA represents the promoter region of the cutinase $\mathrm{A}$ gene from $B$. cinerea. uidA $(\beta$ glucuronidase) and $h p h$ (hygromycin phosphotransferase) are Escherichia coli genes, Trp C and PoliC are terminator and promoter sequences, respectively, from Aspergillus nidulans. Arrows indicate the orientation of the fragment. Small arrowheads above the boxes represent primers used for PCR. RI $=E c o \mathrm{RI}, \mathrm{RV}=E c o \mathrm{RV}, \mathrm{HIII}=H i n d I I I$. Restriction sites between brackets were deleted during subcloning.

Table 1. GUS activity in mycelia of transformants $\mathrm{T} 2$ and $\mathrm{T} 7$ and the wild-type strain SAS56a

\begin{tabular}{lccc}
\hline Day & T2 & T7 & SAS56 \\
\hline 0 & 0.95 & 0.39 & 0.04 \\
1 & 11.89 & 5.45 & 0.07 \\
2 & 12.85 & 6.38 & 0.06 \\
3 & 9.60 & 1.56 & 0.02 \\
\hline
\end{tabular}

${ }^{a}$ At day 0 the cutin monomer 16-hha was added. Units of GUS activity are defined in nmol 4-methyl umbelliferone produced $/ \mathrm{h} / 10 \mu \mathrm{g}$ protein. Data of one experiment are shown and were reproducible in a second independent experiment. ity ( $<1$ unit) was detected in transformants $\mathrm{T} 2$ and $\mathrm{T} 7$ at day 0 . In both transformants GUS activity increased on days 1 and 2 after addition of 16-hha (Table 1) and it decreased again on day 3. In the recipient strain SAS56 lacking the pCutGUS construct, no significant GUS activity was detected (Table 1).

To investigate in closer detail whether uidA mRNA and cutA mRNA are expressed similarly from the cutA promoter under different cutinase inducing and repressing conditions (van der Vlugt-Bergmans et al. 1997), expression of the genes was also studied by RNA blot analysis. Transformants T2 and T7 were grown in a liquid culture for 2 days and subsequently diluted (day 0) in three different media: medium A contained 16-hha, medium B contained 16-hha and glucose and in medium $\mathrm{C}$ only glucose was present. The glucose concentration in media $\mathrm{B}$ and $\mathrm{C}$ decreased to undetectable levels between day 1 and day 2 after transfer of the mycelium.

Mycelia from the start culture and media A to $\mathrm{C}$ were sampled at day 0 and day 1 to 3 , respectively, for isolation of RNA. Results of the RNA blot analysis are shown in Figure 3. Both cutA and uidA transcripts of 900 and 2,000 nt, respectively, were detected from day 1 onwards when mycelium was grown in the presence of 16-hha (medium A). In medium B, transformant T2 expressed cutA and uidA transcripts at days 2 and 3 when glucose had been depleted from the medium, indicating that glucose repression of the cutA promoter affects the expression of the uidA gene as well. In the absence of the inducer (T2, medium C), only weak hybridization signals of cutA and uidA were present. Hybridization of the blot with a

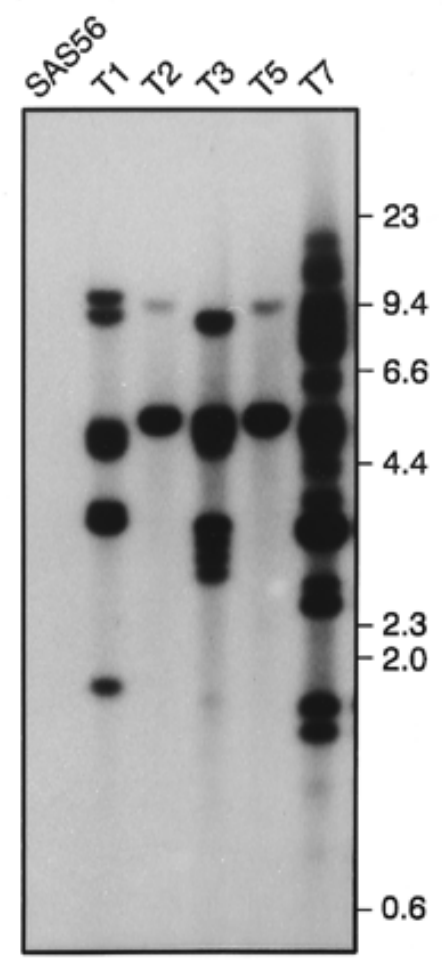

Fig. 2. Autoradiograph of a Southern blot containing genomic DNA of Botrytis cinerea wild-type strain SAS56 and the transformants T1, T2, T3, T5 and T7, digested with EcoRV. The blot was hybridized with the EcoRV/HindIII fragment of pCutGUS (Fig. 1A), containing parts of the uidA coding region and trpC terminator. Molecular size markers are indicated in $\mathrm{kbp}$ in the right margin. 
probe derived from the constitutively expressed $\beta$-tubulin gene of B. cinerea (tubA, accession number Z69263 of the GenEMBL database) showed similar amounts of fungal RNA in each lane on the blot. Co-expression of cutA and uidA was also observed in transformant T7, except for the samples harvested at day 1 (medium B) and days 2 and 3 (medium C). Moreover, hybridization of the blot containing T7 RNA with the uidA probe shows an additional larger transcript, which is coregulated with the 2,000 nt uidA transcript. Since the pCutGUS construct contained a terminator sequence upstream of the cutA promoter, this transcript probably results from termination downstream of the $\operatorname{trpC}$ terminator. Smears are observed below the uidA transcript and the hybridization signal decreases compared to that of cutA at day 3 (media A and B), suggesting that uidA mRNA is more rapidly turned over than cutA mRNA. Nevertheless it can be concluded from these analyses that the cutA gene and the uidA gene under control of the $c u t A$ promoter are synchronously induced in transformants $\mathrm{T} 2$ and $\mathrm{T} 7$. The induction by 16-hha and repression by glucose are consistent with the results described in the accompanying paper (Van der Vlugt-Bergmans et al. 1996).

\section{Histochemical staining of GUS activity.}

Conidia of both transformants $\mathrm{T} 2$ and $\mathrm{T} 7$, harvested from plates, did not contain any detectable GUS activity. When conidia were germinated on water agar containing X-Gluc, a pale blue colony was obtained. Addition of a suspension of 16-hha to the agar yielded colonies with a more intense blue color. When the conidia were germinated on X-Gluc plates
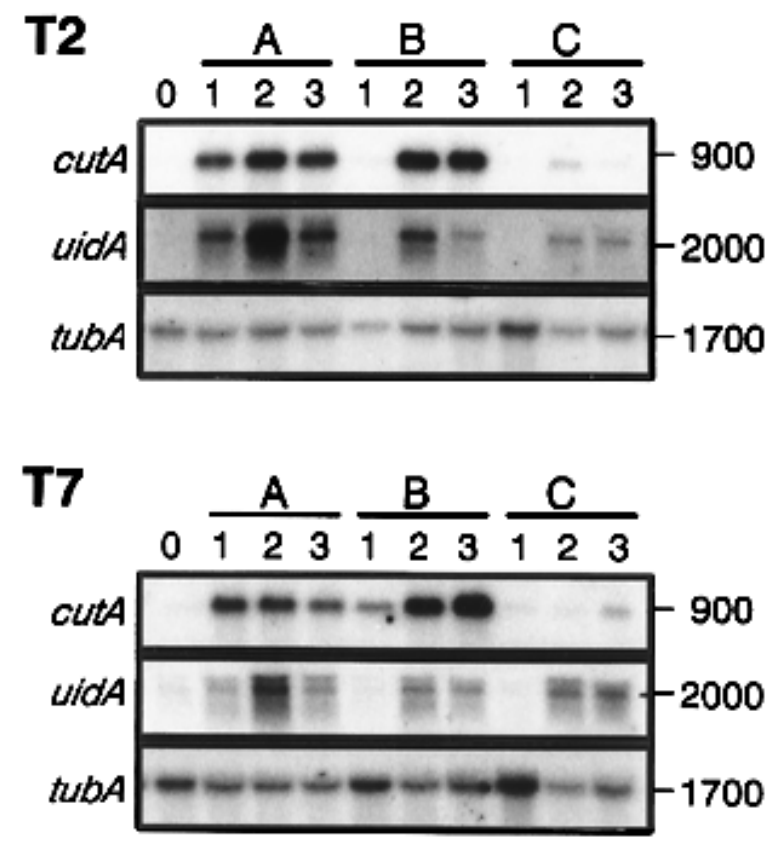

Fig. 3. Autoradiograph of an RNA blot containing total RNA of Botrytis cinerea transformants T2 and T7, grown under different conditions. Mycelium from a liquid start culture (lane 0 ) was diluted into fresh medium containing 16-hydroxyhexadecanoic acid (A), 16-hydroxyhexadecanoic acid and glucose (B), or only glucose (C), and harvested after 1, 2 and 3 days, as indicated above the lanes. The blot was hybridized with probes containing part of the coding regions of the $c u t \mathrm{~A}$, uid $\mathrm{A}$ and $t u b \mathrm{~A}$ genes. Sizes of the transcripts are indicated in $n t$ in the right margin. containing sucrose the resulting colonies were colorless (results not shown).

The activity of the cutA promoter during penetration of gerbera flowers and tomato fruits was examined in transformants $\mathrm{T} 2$ and $\mathrm{T} 7$ by monitoring GUS activity microscopically in a histochemical assay using X-Gluc as substrate. Both T2 and T7 were able to infect the hosts with the same timing and caused similar symptoms as the recipient strain SAS56. On gerbera flowers dry-inoculated with conidia of $B$. cinerea, necrotic lesions were clearly visible $24 \mathrm{~h}$ postinoculation (h.p.i.). Penetration of the cutin layer had occurred before that timepoint and, therefore, flower petals were harvested and examined for GUS activity at 6, 16 and 24 h.p.i.. At 6 h.p.i., several conidia showed a dark blue color, whereas other conidia were pale blue or colorless (Fig. 4A). At 16 h.p.i., nearly all conidia had germinated and were stained blue. Infection pegs were also stained dark blue (Fig. 4B). Penetration of host cells was observed and at 24 h.p.i. cell death was visible by yellowbrownish cell contents of penetrated epidermal cells (Fig. 4C). In SAS56, germination and penetration were observed with equal efficiency and timing. However, no blue staining was observed at any time during infection with SAS56 (not shown).

On tomato fruits $B$. cinerea developed more slowly than on gerbera flowers. Two days postinoculation (d.p.i.) $60 \%$ of the conidia had germinated and $40 \%$ of the total number of conidia had penetrated into epidermal cells. All fungal structures, except for some ungerminated conidia, were stained dark blue and penetration of host tissue was visible (Fig. 4D). At 3 d.p.i., up to $90 \%$ of the conidia had germinated and $50 \%$ had penetrated. Death of single epidermal cells was visible in the microscope, although no necrotic lesions were seen by eye.

\section{Characterization of cutinase A-deficient transformants.}

To determine whether cutinase A is essential for successful penetration of host tissue by $B$. cinerea, the single copy cutA gene was mutated by gene disruption. Transformation vector pGDcut 2 containing a hygromycin B selection marker $(h p h)$ flanked by promoter and terminator sequences of cutA (PcutA and TcutA; Fig. 1B), was used to transform the haploid B. cinerea strain B05.10. Pathogenicity of B05.10 was indistinguishable from strain SAS56 from which it was derived (P. Büttner and P. Tudzinsky, unpublished). Homologous recombination between the cutA locus and cutA sequences present in pGDcut 2 would allow replacement of the wild-type gene by the hygromycin cassette.

Eighty hygromycin B resistant colonies obtained after transformation of strain B05.10 with pGDcut 2 were screened by PCR using primers complementary to cutA sequences which were deleted in pGDcut2 (Fig. 1B). A 400-bp PCR fragment was predicted from the wild-type cutA locus, whereas no amplification product was expected in transformants in which gene disruption had occurred. In 23 of the 80 transformants the 400-bp fragment was absent or only weakly amplified, as compared to the intensity of the amplification product in other transformants. Six of the 23 transformants were selected for further analysis by Southern blotting (Fig. 5A). Genomic DNA digested with HindIII was predicted to release a $2.1-\mathrm{kb}$ fragment from the wild-type cutA gene or a $4.0-\mathrm{kb}$ fragment from the mutated gene (Fig. 1B). Using a HindIII/SstI fragment of the cutA promoter (see Fig. 1B) as probe, a $4.0-\mathrm{kb}$ 
fragment was detected in all six transformants indicating that homologous recombination had occurred at the cutA locus. However, in three transformants (T124, T125, and T137) the wild-type $2.1-\mathrm{kb}$ fragment also weakly hybridized, indicating that the wild-type cutA gene is still present in these transformants. Nonpredicted hybridization signals in T137 (Fig. 5A) demonstrate that ectopic vector integration had also occurred. Hybridization of the same blot with a HindIII/EcoRI fragment of the hygromycin cassette (Fig. 1B) showed only hybridization of the 4.0-kb fragment in T124, T125, T130, T132, and $\mathrm{T} 136$, and the same additional fragments of T137 as detected in Fig. 5A (results not shown). Since no ectopic integrations of the selection marker were detected in T124 and T125, a small proportion of untransformed nuclei harboring the wildtype cutA gene is apparently still present in these tranformants.
To monitor cutA transcript levels in the recipient strain and transformants, RNA blot analysis was performed on total RNA isolated from transformants grown in cutinase inducing medium (Fig. 5B). A single band of $900 \mathrm{nt}$ was detected in B05.10 and, to a lesser extent, in transformants T124, T125 and T137, in which the wild-type cutA gene was also detected by Southern blotting (see Fig. 5A). No cutA mRNA was detected in transformants T130, T132, and T136. Cohybridization with a probe derived from the constitutively expressed $\beta$ tubulin gene tubA of $B$. cinerea demonstrated the presence of approximately equal amounts of RNA on the blot.

Southern and RNA blot data demonstrate that the cutA gene is not expressed in transformants T130, T132, and T136. Viability of transformants T130, T132 and the recipient strain B05.10 was tested by plating conidia on solid medium. After 2 days of incubation $90 \%$ of T130 or T132 conidia had germi-
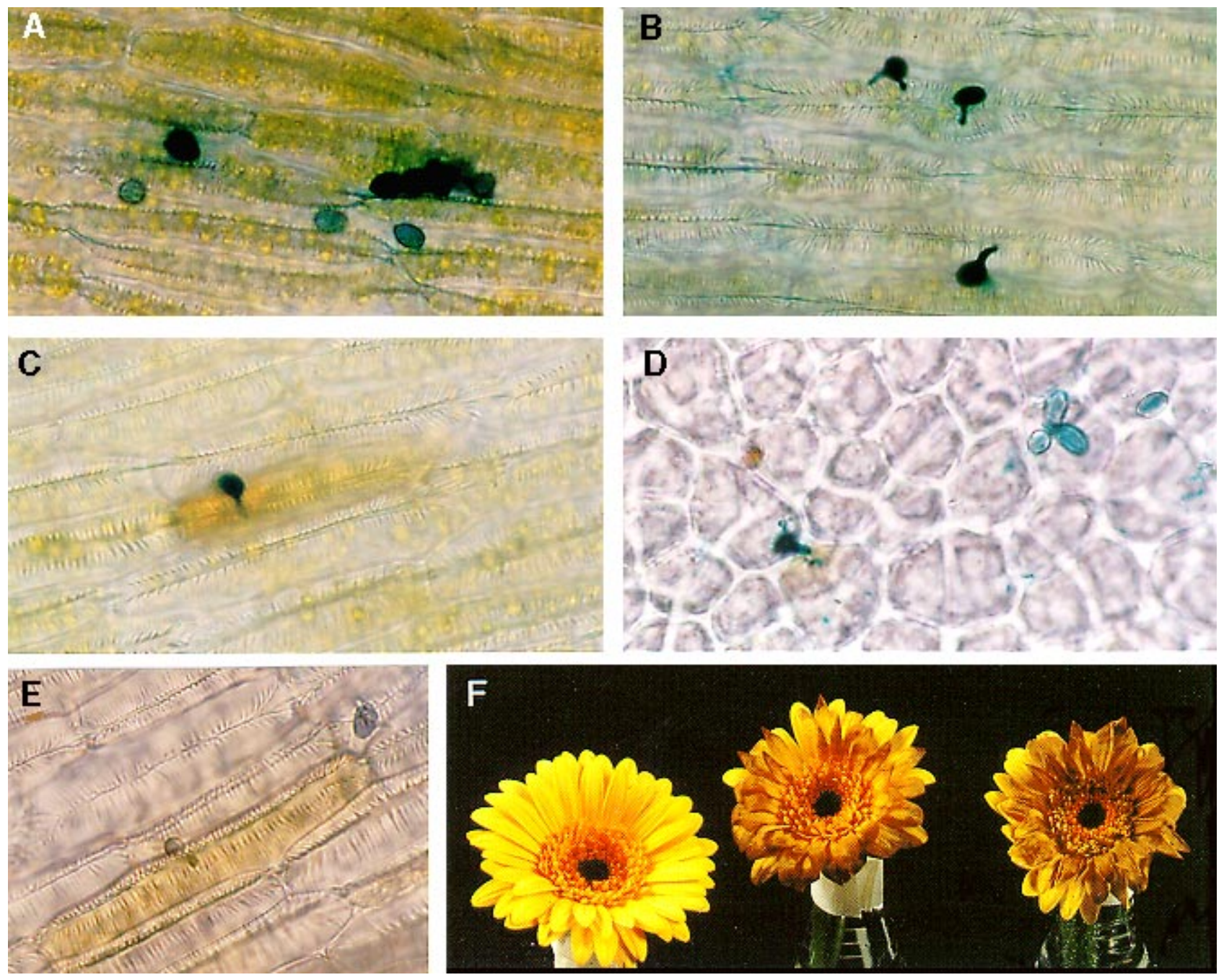

Figure 4. Histochemical localization of GUS activity on gerbera petals (A-C) and tomato fruit (D) inoculated with transformant T2 of Botrytis cinerea containing the cutA promoter-GUS fusion. A, Conidia on a gerbera petal showing different levels of GUS activity at 6 h.p.i.. B, At 16 h.p.i. most conidia have germinated and show high GUS activity in conidia and infection pegs. C, At 24 h.p.i. entry of an infection peg into an epidermal cell has led to cell death visualized by yellowish-brown cell content. D, Three d.p.i. on tomato fruit, conidia show different levels of GUS activity and one germinated conidium has penetrated an epidermal cell. E, Conidia of transformant T130 lacking a functional cutinase A gene, germinate and penetrate epidermal cells of gerbera petals as well as the wild-type SAS56. Fungal structures are stained with lactophenol-cotton blue dye. Note the yellowish-brown cell content of the penetrated epidermal cell. F, Gerbera flowers noninoculated (left) and inoculated with B05.10 (middle) and T130 (right). 
nated compared to $100 \%$ germination in B05.10. All germinated conidia developed into normal colonies. One of the mutants was further characterized biochemically. Recipient strain B05.10 and the mutant T132 were grown in liquid culture containing tomato cutin for 4 weeks. The culture filtrates were partially purified by acetone precipitation and gel filtration chromatography. Fractions containing PNB hydrolytic activity were pooled and analysed on a chromatofocusing column with a $\mathrm{pH}$ gradient of 7.4 to 5 (Fig. 6). The recipient strain B05.10 contained PNB hydrolytic acitivity in two peaks, eluting at $\mathrm{pH} 6.3$ and 5.75. The latter peak corresponds to the cutinase A (van der Vlugt-Bergmans et al. 1997). Mutant T132 contained PNB hydrolytic activity, but none of this activity eluted at pH 5.75 (Fig. 6).

\section{Pathogenicity assay of cutinase A-deficient mutants.}

Conidia of recipient strain B05.10 and transformants T130 and T132 (lacking the cutA gene) were dry-inoculated on
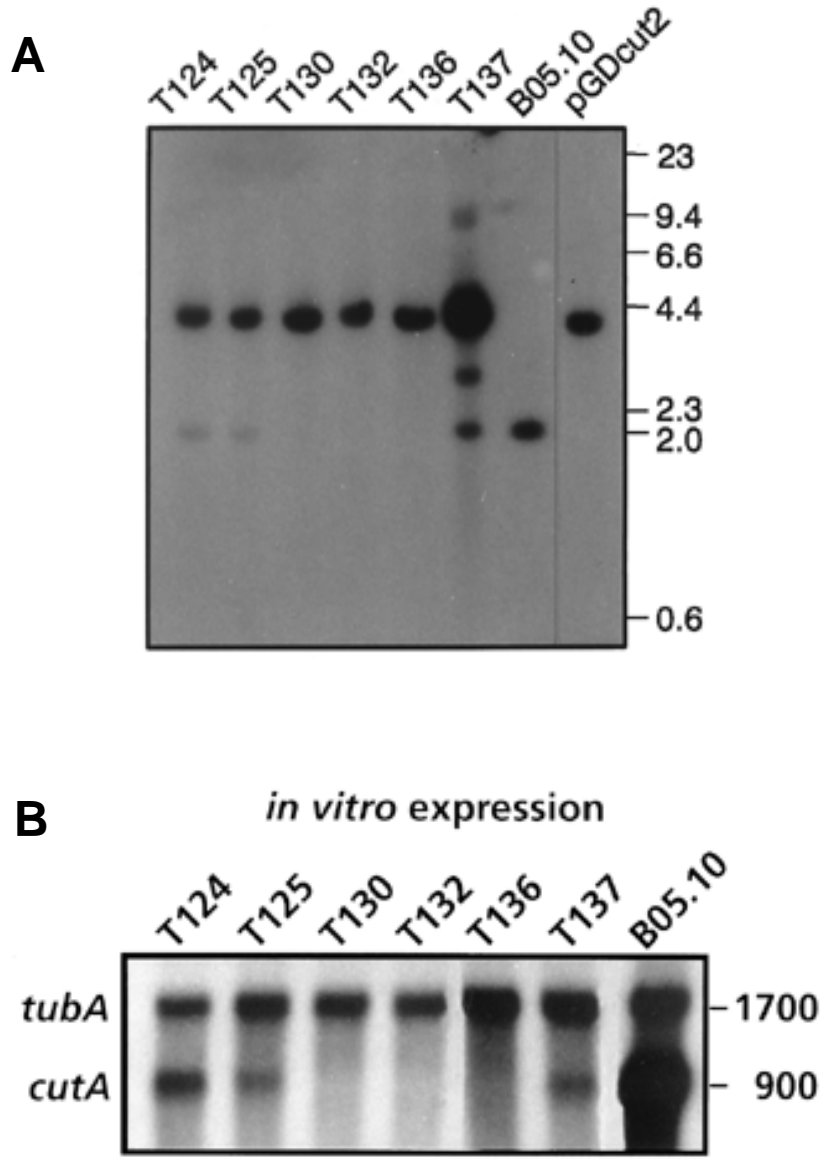

Fig. 5. A, Autoradiograph of a Southern blot containing genomic DNA of Botrytis cinerea wild-type strain B05.10 and the transformants T124, T125, T130, T132, T136, T137, and the gene disruption plasmid pGDcut2 digested with HindIII. The blot was hybridized with the Hin$\mathrm{dIII} /$ SstI fragment of pGDcut2 (Fig. 1A), containing a part of the cutA promoter sequence. Molecular-size markers are indicated in kbp in the right margin. B, Autoradiograph of a Northern blot containing total RNA of B. cinerea wildtype strain B05.10 and the transformants T124, T125, T130, T132, T136, T137, isolated from mycelium induced for $c u t A$ expression. As probe parts of the cutA and $t u b A$ encoding regions of these $B$. cinerea genes have been used. Sizes of the transcripts are shown in nt at the right margin. flowers of gerbera cv. Sirtaki and on tomato fruits. Symptoms on gerbera flowers were first visible 24 h.p.i. as necrotic lesions occurring everywhere on the petals. At 48 h.p.i. flowers were completely rotten (Fig. 4F). Penetration of epidermal gerbera cells was examined microscopically by cotton blue staining of fungal hyphae in inoculated tissue. No morphological differences were observed among the fungal penetration structures of B05.10, T130 and T132. Penetration by all strains occurred via epidermal cells and all strains caused death of host cells, as indicated by yellow-brownish stained cell contents (Fig. 4E). Since the structure and thickness of the cuticle may vary with plant age or cultivar (Martin and Juniper 1970), gerbera flowers of different age and of a different cultivar ('Romana') were tested. Also on these flowers, no differences in pathogenicity of T130 and T132 as compared to B05.10 were observed.

On tomato fruits no necrotic lesions were visible on the fruit surface. At 3 d.p.i. the interior of the fruit was colonized resulting in complete maceration of the tissue 5 d.p.i. In two independent tests on two gerbera flowers and on tomato fruits, no significant differences in lesion size, lesion number, or rate of disease development were observed between B05.10 and the cutinase A-deficient transformants T130 and T132. B. cinerea was reisolated from tomato fruits inoculated with B05.10, T130, and T132 and analyzed by PCR using cutAspecific primers (Fig. 1B). A 0.4-kb fragment was amplified from the strain, reisolated from tomato fruits inoculated with B05.10. This fragment was not amplified in PCR assays using strains reisolated from tomato fruits inoculated with T130 and T132 (result not shown). This demonstrates that the strains obtained from the tomato fruit inoculated with mutant strains $\mathrm{T} 130$ and $\mathrm{T} 132$ had retained the cutA deletion.

\section{DISCUSSION}

Cutinase was suggested to play an important role in penetrating host tissue by $B$. cinerea. This was based on electron microscopy studies (McKeen 1974; Rijkenberg et al. 1980) and on the observation that monoclonal antibodies raised against cutinase A reduced lesion formation by $B$. cinerea on

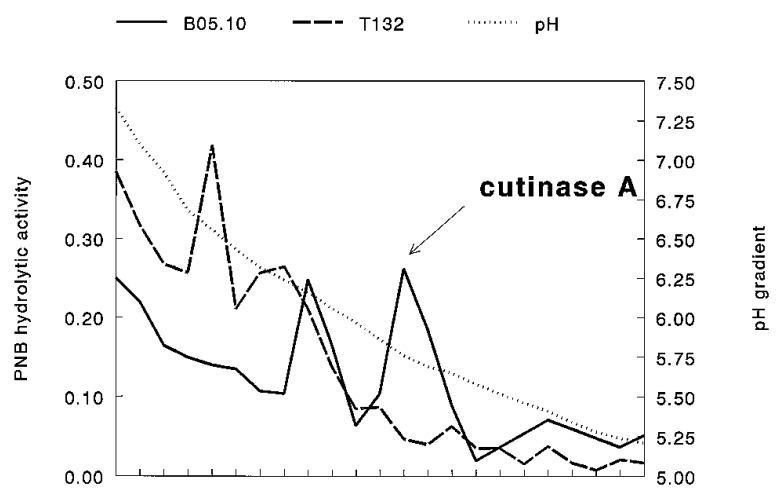

Fig. 6. Biochemical characterization of cutA mutant T132. Culture filtrates of strain B05.10 and transformant T132, grown in liquid culture containing tomato cutin, were precipitated with acetone, fractionated by gel filtration and subjected to chromatofocusing. In each fraction, the $\mathrm{pH}$ and the PNB hydrolytic activity $\left(\Delta \mathrm{A}_{405} / \mathrm{h}\right)$ were determined. Solid line: strain B05.10. Dashed line: transformant T132. Dotted line: profile of the $\mathrm{pH}$ gradient. 
gerbera flowers (Salinas 1992). However, proof for production of cutinase $\mathrm{A}$ at the site of penetration was lacking and, therefore, its role remained unclear. In this study the expression of cutinase A during penetration of host tissue by $B$. cinerea was analyzed by using transformants with the GUS reporter gene uidA under control of the $c u t A$ promoter. The requirement for cutinase $\mathrm{A}$ in the penetration process was investigated by using cutinase A-deficient mutants obtained by gene disruption in pathogenicity assays.

Transformants of $B$. cinerea containing the cutA promoterGUS fusion construct were first tested in vitro by GUS activity assays and RNA blot analysis, in order to select transformants in which uidA and cutA mRNAs are similarly expressed from the cutA promoter. X-Gluc plate assays with transformants T2 and T7 provided further evidence for the activation of the cutA promoter by the cutin monomer 16-hha and for catabolite repression by glucose (van der Vlugt-Bergmans et al. 1997). These two transformants were used in histochemical analysis of GUS activity during penetration of host tissue. On gerbera petals and epidermal strips of tomato fruits inoculated with $\mathrm{T} 2$ and $\mathrm{T} 7$, intense blue staining was observed from the onset of conidial germination and in penetration pegs. Without exception, conidia that had germinated stained blue. Thus cutinase A expression starts during germination, just prior to the penetration event, in which the enzyme was proposed to play a role. GUS activity could not be followed throughout the infection of the gerbera flowers or the tomato fruits because the plant tissues were severely macerated at later stages of the infection, thus hampering histochemical staining techniques. However, the observation that cutA mRNA is expressed in $B$. cinerea-infected tomato leaves until the stage of sporulation (van der Vlugt-Bergmans et al. 1997) suggests that cutinase A is not only produced in early stages of the infection process. The pCutGUS transformants were not tested on tomato leaves. In this bioassay the addition of glucose in the inoculum is essential for the initiation of infection and glucose was shown to repress the cutA gene (Van der Vlugt-Bergmans et al. 1997).

The requirement of cutinase A for enzyme-mediated penetration was investigated by using cutinase A-deficient mutants, constructed by replacement of cutA coding sequences with a hygromycin marker. Southern blot analysis of six transformants showed three transformants lacking the wild-type cutA gene and three transformants containing both the disrupted and wild-type cutA gene. The latter gene was present in much lower abundance as shown by weaker hybridization signals. Since the selection marker was not ectopically integrated in transformants T124 and T125, the wild-type cutA gene is probably present in nuclei which are hygromycin B sensitive and thus untransformed. Due to the multinucleate, heterokaryotic nature of $B$. cinerea (van der Vlugt-Bergmans et al. 1993), transformed and untransformed nuclei can coexist in one thallus. Apparently, untransformed nuclei survived hygromycin B selection due to the presence of resistant nuclei in transformants $\mathrm{T} 124$ and T125. The presence of both types of nuclei hampered the initial detection of gene disruption by PCR, since the PCR assay was based on absence or presence of an amplification product of the cutA gene. In 23 out of 80 transformants the PCR fragment was weakly amplified or completely absent. Southern blot analysis of 6 out of these 23 showed gene disruption in all six transformants. This suggests that homologous recombination had occurred with a frequency of approximately $25 \%$ (23:80). However, the actual frequency of cutinase A-deficient mutants is lower due to the presence of untransformed nuclei. In future gene disruption experiments, single spore cultures of $B$. cinerea transformants will be analyzed in order to reduce the number of heterokaryons containing wild-type nuclei. However, this will not guarantee that such heterokaryons are not obtained, because conidia of $B$. cinerea can contain up to 10 nuclei (Shirane et al. 1988).

Transformants T130, T132, and T136 lacked a functional cutA gene, since no cutA mRNA was detected by RNA blot analysis. Pathogenicity tests were conducted with the cutinase A-deficient transformants $\mathrm{T} 130$ and $\mathrm{T} 132$ and the recipient strain B05.10 by inoculation on gerbera flowers and tomato fruits. Both T130 and T132 induced disease symptoms indistinguishable from B05.10 which carries a functional cutA gene. The use of flowers of different age or a different gerbera cultivar, of which the cuticle might be of a different structure or thickness (Martin and Juniper 1970), did not result in altered symptoms. Microscopic examination at early stages of the infection showed that penetration of single germinating conidia of T130 into epidermal cells was similar to the wildtype strain. Thus, cutinase A-deficient mutants of $B$. cinerea show no altered penetration ability and cutinase A is not essential for successful penetration of undamaged host tissue. In contrast, Salinas (1992) observed that treatment of gerbera flowers with monoclonal antibodies raised against cutinase A of B. cinerea strain $\mathrm{Bc} 7$ reduced lesion formation by $80 \%$. The discrepancy between these observations cannot easily be explained, but the results with the cutinase A-deficient mutants exclude a requirement of cutinase A for penetration.

This is the first report of successful gene disruption in $B$. cinerea as a means of specifically mutating a single defined locus, the cutA gene. Cutinase gene disruption in Magnaporthe grisea (Sweigard et al. 1992) and Alternaria brassicicola (Yao and Köller 1995) also did not alter the penetration ability of the fungus. In $M$. grisea, significant remnant cutinase activity was detected in the disruption mutant (Sweigard et al. 1992). In A. brassicicola the cutinase gene that had been disrupted was not expressed in planta to detectable level, and a low level of additional cutinase activity was produced specifically during the penetration of host tissue (Köller et al. 1995; Yao and Köller 1995). There is still controversy over the requirement of a cutinase gene of $F$. solani f.sp. pisi for pathogenicity on pea (Rogers et al. 1994; Stahl et al. 1994).

To our knowledge, the analysis of cutinase gene expression in a plant pathogenic fungus in single penetrating germ tubes by using reporter gene fusions has not been described before. In other pathosystems droplet inoculations with conidial suspensions were performed (Sweigard et al. 1992; Rogers et al. 1994; Stahl et al. 1994; Köller et al. 1995; Yao and Köller 1995). This makes it difficult to exclude side effects of clusters of conidia on plant surfaces. In our gerbera inoculation assays, cutinase A expression was observed in individual conidia that germinated on and penetrated into the host cuticle. In spite of the fact that single conidia were able to penetrate and cause disease, the deletion of the cutA gene did not affect pathogenicity of $B$. cinerea to any detectable extent. Thus, $B$. cinerea apparently possesses other means of penetrating the host surface. In this respect, the production of unrelated cutinases or other enzyme activities during the infection process will have to be investigated. 


\section{MATERIALS AND METHODS}

\section{Fungal growth.}

Strain SAS56 of $B$. cinerea (van der Vlugt-Bergmans et al. 1993), haploid strain B05.10 (derived from SAS56, a gift from P. Büttner and P. Tudzynski, Institut für Botanik, Westfälische Wilhelms-Universität, Münster, Germany), and resulting transformants were grown on malt extract agar (Oxoid, Basingstoke, England) at $18^{\circ} \mathrm{C}$ in the dark. Prior to plant inoculations strains were grown on tPDA (potato dextrose agar containing $300 \mathrm{~g}$ of homogenized tomato leaves per litre). After 3 days the cultures were exposed for $16 \mathrm{~h}$ to near UV-light. One week later conidia were collected from sporulating cultures and inoculated ( $10^{8}$ conidia per liter) in Gamborg's B5 medium (Duchefa Biochemie BV, Haarlem, The Netherlands) supplemented with $0.3 \%$ glucose. After 2 days of growth in a rotary shaker at $160 \mathrm{rpm}, 20^{\circ} \mathrm{C}$, mycelium was harvested for isolation of genomic DNA. For analysis of gene expression, 2day-old cultures (start cultures) were diluted tenfold in the following media: B5 medium $+0.05 \%$ 16hydroxyhexadecanoic acid (16-hha; A), B5 medium $+0.05 \%$ 16 -hha $+0.3 \%$ glucose $(B)$, B5 medium $+0.3 \%$ glucose $(C)$. Remaining mycelium of the start cultures was harvested. All three subcultures were incubated at $20^{\circ} \mathrm{C}, 160 \mathrm{rpm}$ and sampled during the following days. The glucose concentration in liquid culture media was determined according to the method of Nelson (1944).

\section{Construction of transformation vectors.}

Vector pCutGUS containing a fusion between the cutA promoter of $B$. cinerea and the GUS reporter gene uidA was constructed in the following way. A NcoI site (CCATGG) was introduced at the ATG start codon of the cutA gene by PCR using a primer complementary to a region upstream of the ATG (5'-CTCCATGGTGAAATTGATGTATTGATATTTTG$3^{\prime}$ ) in combination with an M13 primer at the $5^{\prime}$-end of the promoter on a subclone containing part of the cutA gene as template. The resulting 1.5-kb PCR fragment was ligated in pBluescript SK (Stratagene) digested with EcoRV. Plasmid pCF20 (Van den Ackerveken et al. 1994) contained the gpd promoter of Aspergillus nidulans, the Escherichia coli uidA gene encoding $\beta$-glucuronidase (GUS, Jefferson et al. 1987) and the terminator of the A. nidulans trpC gene. The gpd promoter was excised from pCF20 by digestion with EcoRI and $N c o I$ and replaced by the cutA promoter present on a $1.5-\mathrm{kb}$ EcoRI/NcoI fragment excised from the Bluescript construct. In this construct, the EcoRI site (upstream of the cutA promoter) was made blunt with the Klenow fragment of DNA polymerase and a $0.7-\mathrm{kb} E c o \mathrm{RV}$ fragment containing the terminator sequence of tubA gene of B.cinerea (TtubA) was inserted (Fig. 1A). The correct orientation of the tubA terminator in vector pCutGUS was checked by PCR using one of the primers complementary to $5^{\prime}$ - and $3^{\prime}$-sequences of the tubA terminator in combination with a primer in the uidA gene (Fig. 1A).

Vector pGDcut2 was derived from plasmid pCut1 (van der Vlugt-Bergmans et al. 1997). The main part of the coding sequence of cutA was deleted by digestion with EcoRV and replaced by a selection marker digested from plasmid pOHT (Hilber et al. 1994; provided by M. Ward, Genencor International, San Francisco, CA). The selection marker was present on a 2.6-kb SmaI/HindIII fragment of pOHT and consisted of the bacterial hygromycin phosphotransferase gene ( $h p h$, conferring resistance to hygromycin $\mathrm{B}$ ) fused to the oliC promoter and $\operatorname{trpC}$ terminator of $A$. nidulans. This fragment was incubated with the Klenow fragment of DNA polymerase to fill in the ends and used to replace the cutA EcoRV fragment from plasmid pCut1 (Fig. 1B). The orientation of the hygromycin B selection marker in the resulting pGDcut 2 construct was determined by restriction analysis.

\section{Transformation of $\boldsymbol{B}$. cinerea.}

Conidia from sporulating cultures of $B$. cinerea strain SAS56 or B05.10 were collected and inoculated in bacto malt extract (Difco, $10^{5}$ conidia/ml). Cultures were incubated in a rotary shaker at $150 \mathrm{rpm}$ at $22^{\circ} \mathrm{C}$ for 2 days. The following transformation protocol was adapted from Hamada et al. 1994. Mycelium was harvested by filtration and washed twice in sterile water and once in $0.6 \mathrm{M} \mathrm{KCl}, 50 \mathrm{mM} \mathrm{CaCl} 2(\mathrm{KC}$ buffer). Protoplasts were generated by adding $10 \mathrm{ml}$ of a filtersterilized solution containing $5 \mathrm{mg}$ of Novozyme 234 (Novolabs) in KC-buffer per gram of fresh weight mycelium. This suspension was incubated for $2 \mathrm{~h}$ at $23^{\circ} \mathrm{C}$ with shaking at $80 \mathrm{rpm}$. The digested mycelium was filtered using filters with 25 and $10 \mu \mathrm{m}$ mesh size, respectively. Protoplasts were pelleted by centrifugation at $2,000 \times g$ for $10 \mathrm{~min}$, washed once and resuspended in KC-buffer to a final concentration of $10^{8}$ protoplasts per ml. Portions of $100 \mu \mathrm{l}$ were maintained on ice for $5 \mathrm{~min}$ and, subsequently, plasmid DNA in $40 \mu \mathrm{l}$ of water, 5 $\mu \mathrm{l}$ of $50 \mathrm{mM}$ spermidine and $100 \mu \mathrm{l}$ of $25 \%$ PEG 3350 (Sigma) in $50 \mathrm{mM} \mathrm{CaCl}_{2}, 10 \mathrm{mM}$ Tris-HCl, $\mathrm{pH} 7.5$, were, one by one, gently added to the protoplasts. The mixture was kept on ice for $20 \mathrm{~min}$. Five-hundred microliters of PEG solution were added, gently mixed and incubated for $10 \mathrm{~min}$ at room temperature. Finally, the volume was adjusted to $1 \mathrm{ml}$ with KC-buffer. For selection, $100 \mu \mathrm{l}$ of protoplasts were spread on $10 \mathrm{ml}$ of SH-agar (0.6 M sucrose, $5 \mathrm{mM}$ HEPES, pH 5.3, 1 $\mathrm{mM}\left(\mathrm{NH}_{4}\right)_{2} \mathrm{HPO}_{4}, 1.2 \%$ agar) and incubated for $3 \mathrm{~h}$ at room temperature. Protoplasts were then overlayed with $3 \mathrm{ml}$ of SH-top agar (0.6\% agar) containing $100 \mu \mathrm{g} / \mathrm{ml}$ of hygromycin $\mathrm{B}$ (Duchefa). After 3 to 7 days of incubation at $22^{\circ} \mathrm{C}$, hygromycin B-resistant colonies of $B$. cinerea were individually transferred to malt extract agar and grown for 2 days at $22^{\circ} \mathrm{C}$. Agar plugs with mycelium from the edge of each colony were subsequently transferred to malt extract agar amended with $100 \mu \mathrm{g} / \mathrm{ml}$ hygromycin B.

Prior to transformation of $B$. cinerea strain SAS56, vector pCutGUS was linearized with $S p h$ I (Fig. 1A) and pOHT with HindIII. Ten micrograms of linearized vector and $1 \mu \mathrm{g}$ of linearized pOHT as selection marker were used for cotransformation. The haploid strain B05.10 of B. cinerea was transformed using $2 \mu \mathrm{g}$ of circular pGDcut 2 .

\section{Southern blot analysis.}

Genomic DNA was isolated according to Drenth et al. (1993), digested with restriction enzymes, size separated on a $0.7 \%$ agarose gel and blotted onto Hybond- $\mathrm{N}^{+}$(Amersham) according to Sambrook et al. (1989). The blot was hybridized in modified Church buffer $(0.5 \mathrm{M}$ sodium phosphate buffer, $\mathrm{pH} 7.2$ and $7 \%$ SDS; Church and Gilbert 1984) at $65^{\circ} \mathrm{C}$ for 18 $\mathrm{h}$ in the presence of a random-primed $\left[\alpha{ }^{3} \mathrm{P}\right] \mathrm{dATP}$ labeled probe. Blots were washed in $0.2 \times \mathrm{SSC}$ and $0.5 \% \mathrm{SDS}$ at $65^{\circ} \mathrm{C}$ and exposed to Kodak X-OMAT AR film. 


\section{RNA blot analysis.}

Total RNA was isolated from mycelial samples using the Extract-A-Plant RNA kit (Clontech). Samples of $10 \mu \mathrm{g}$ total RNA were denatured with formamide and formaldehyde and subjected to electrophoresis on a $1.0 \%$ agarose gel containing formaldehyde (Sambrook et al. 1989). After blotting to Hybond- $\mathrm{N}^{+}$membranes, blots were hybridized as described above in the presence of a random-primed $\left[\alpha-{ }^{32} \mathrm{P}\right] \mathrm{dATP}$ labelled probe and washed in $0.2 \times \mathrm{SSC}$ and $0.5 \% \mathrm{SDS}$ at $65^{\circ} \mathrm{C}$ efore exposure to Kodak X-OMAT AR film.

\section{Biochemical analysis.}

Recipient strain B05.10 and cutA mutant T132 were grown in $125 \mathrm{ml}$ of liquid medium as described above. After 3 days, $500 \mathrm{mg}$ of tomato cutin was added and the culture was incubated under continuous shaking for 30 days. The culture filtrates were harvested, acetone was added to a final concentration of $20 \%$ and the sample was centrifuged at $2,000 \times g$ at $4^{\circ} \mathrm{C}$ for $20 \mathrm{~min}$. The supernatant was adjusted to $80 \%$ acetone and precipitated proteins were pelleted by centrifugation at $3,000 \times g$ at $4^{\circ} \mathrm{C}$ for $30 \mathrm{~min}$. The pellet was dried, dissolved in $40 \mathrm{mM}$ Tris-HCl, $50 \mathrm{mM} \mathrm{NaCl}, \mathrm{pH} 7.5$ and loaded on a Sephadex $\mathrm{G} 75$ column $(1.5 \times 25 \mathrm{~cm})$ equilibrated with the same buffer. Fractions of $4 \mathrm{ml}$ were collected and measured for PNB hydrolytic acitivity. Fractions containing enzyme activity were pooled, dialyzed, and applied to a PBE 94 column as described in the accompanying paper (van der VlugtBergmans et al. 1997). The $\mathrm{pH}$ and the PNB hydrolytic activity of each fraction were determined.

\section{Fluorometric GUS assay.}

One hundred milligrams of fresh mycelium was resuspended in GUS extraction buffer (GEB: $50 \mathrm{mM}$ sodium phosphate buffer, $\mathrm{pH} 7.0,10 \mathrm{mM}$ EDTA, $0.1 \%$ N-laurylsarcosine, $0.1 \%$ Triton $\mathrm{X}-100,10 \mathrm{mM} \beta$-mercaptoethanol; Jefferson et al. 1987), ground with a pestle, vortexed and after centrifugation the supernatant was stored at $4{ }^{\circ} \mathrm{C}$. The protein concentration was determined using the Bio-Rad protein assay (Bradford 1976) and bovine IgG as a standard. Ten micrograms of protein of each sample was diluted to $100 \mu$ in GEB and added to $500 \mu \mathrm{l}$ of $1 \mathrm{mM}$ MUG (4-methyl umbelliferyl glucuronide, Research Organics) dissolved in GEB. After mixing, enzyme assays were incubated at $37^{\circ} \mathrm{C}$ in the dark. After 1 and $2 \mathrm{~h}$ of incubation, $100 \mu \mathrm{l}$ of each enzyme assay was mixed with $900-\mu \mathrm{l}$ stop buffer $\left(0.2 \mathrm{M} \mathrm{Na}_{2} \mathrm{CO}_{3}\right)$. Fluorescence was measured in a spectrofluorimeter (excitation 365 $\mathrm{nm}$, emission $450 \mathrm{~nm}$ ), calibrated with MU (4-methyl umbelliferone, Sigma) in stop buffer.

\section{Bioassays.}

Gerbera flowers cv. Sirtaki and Romana and tomato fruits cv. Moneymaker (green fruits of 3 to $4 \mathrm{~cm}$ in diameter) were dry-inoculated with transformants or wild-type strains of $B$. cinerea. Conidia were transferred from sporulating cultures by a brush, or they were dusted on the host tissue by tapping the bottom of an inverted petri dish with a sporulating culture.

Flowers and fruits were incubated in closed plastic boxes with a transparent lid to obtain a humidity of $100 \%$, at $20^{\circ} \mathrm{C}$ with a dark-light cycle of $16 \mathrm{~h}$ light and $8 \mathrm{~h}$ dark. Development of disease symptoms was followed by eye and fungal penetration was examined microscopically in whole-mount preparations of inoculated petals or in epidermal strips of tomato fruits. Flower petals harvested at different time-points after inoculation were fixed in absolute alcohol-glacial acetic acid $(1: 1 \mathrm{v} / \mathrm{v})$, cleared in $75 \%$ lactic acid for $48 \mathrm{~h}$ at $37^{\circ} \mathrm{C}$, and mounted on a microscope slide in lactophenol-cotton blue dye. Epidermal strips of tomato fruits were similarly treated with omission of the clearing step.

For histochemical localization of GUS activity, gerbera petals or epidermal strips of tomato fruits were incubated with $0.5 \mathrm{mg} / \mathrm{ml} \mathrm{X-Gluc} \mathrm{(5-bromo-4-chloro-3-indolyl} \beta$-D glucuronide, Biosynth $\mathrm{AG}$ ) in $50 \mathrm{mM}$ phosphate buffer, $\mathrm{pH} 7.0,1$ $\mathrm{mM} \mathrm{KFeCN}$ and $0.05 \%(\mathrm{v} / \mathrm{v})$ Triton-X100, overnight at $37^{\circ} \mathrm{C}$. The tissue was mounted on microscope slides in $50 \%(\mathrm{v} / \mathrm{v})$ glycerol.

The samples were examined with a Zeiss Axioscope microscope and photographs were taken using a Zeiss MC-100 camera unit. Bioassays were performed two times.

Reisolation of mycelium from infected tomato fruit was performed by incubating a piece of infected fruit tissue on water agar at $18^{\circ} \mathrm{C}$. After 3 days of incubation mycelium bearing agar plugs were excised from the edge of the developing colony and incubated on malt extract agar as described above.

\section{ACKNOWLEDGMENTS}

We thank P. Büttner and P. Tudzynski (Institut für Botanik, Westfälische Wilhelms-Universität, Münster, Germany) for providing the haploid strain B05.10 of B. cinerea, and Dr. M. Ward (Genencor International, San Francisco, CA) for providing plasmid pOHT.

\section{LITERATURE CITED}

Baker, C. J., and Bateman, D. F. 1978. Cutin degradation by plant pathogenic fungi. Phytopathology 68:1577-1584.

Bradford, M. 1976. A rapid and sensitive method for the quantitation of microgram quantities of proteins utilizing the principle of protein dye binding. Anal. Biochem. 72:248-250.

Büttner, P., Koch, F., Voigt, K., Quidde, T., Risch, S., Blaich, R., Brückner, B., and Tudzynski, P. 1994. Variations in ploidy among isolates of Botrytis cinerea: implications for genetic and molecular analysis. Curr. Genet. 25:445-450.

Church, G. M., and Gilbert, W. 1984. Genomic sequencing. Proc. Natl. Acad. Sci. USA 81:1991-1995.

Clark, C. A., and Lorbeer, J. W. 1976. Comparative histopathology of Botrytis squamosa and Botrytis cinerea on onion leaves. Phytopathology 66:1279-1289.

Drenth, A., Goodwin, S. B., Fry, W. E., and Davidse, L. C. 1993. Genotypic diversity of Phytophthora infestans in The Netherlands revealed by DNA polymorphisms. Phytopathology 83:1087-1092.

Hamada, W., Reignault, Ph., Bompeix, G., and Boccara, M. 1994. Transformation of Botrytis cinerea with the hygromycin B resistance gene, $h p h$. Curr. Genet. 26:251-255.

Hilber, U. W., Bodmer, M., Smith, F. D., and Köller, W. 1994. Biolistic transformation of conidia of Botryotinia fuckeliana. Curr. Genet. 25:124-127.

Jarvis, W. R. 1977. Botryotinia and Botrytis Species-Taxonomy, Physiology and Pathogenicity — a Guide to Literature. Canada Department of Agriculture, Ottawa.

Jefferson, R. A., Kavanagh, T. A., and Bevan, M. W. 1987. GUS fusions: $\beta$-Glucuronidase as a sensitive and versatile gene fusion marker in higher plants. EMBO J. 6:3901-3907.

Köller, W., Yao, C., Trail, F., and Parker, D. M. 1995. Role of cutinase in the invasion of plants. Can. J. Bot. 73 (suppl. 1): S1109-S1118.

Martin, J. T., and Juniper B. E. 1970. The Cuticles of Plants. St. Marti n's New York.

McKeen, W. E. 1974. Mode of penetration of epidermal cell walls of Vicia faba by Botrytis cinerea. Phytopathology 64:461-467. 
Nelson, N. 1944. A photometric adaptation of the Somogyi method for the determination of glucose. J. Biol. Chem. 153: 375-380.

Rijkenberg, F. H. J., De Leeuw, G. T. N., and Verhoeff, K. 1980. Light and electron microscopy studies on the infection of tomato fruits by Botrytis cinerea. Can. J. Bot. 58:1394-1404.

Rogers, L. M., Flaishman, M. A., and Kolattukudy, P. E. 1994. Cutinase gene disruption in Fusarium solani f.sp. pisi decreases its virulence on pea. Plant Cell 6:935-945.

Salinas, J. 1992. Function of cutinolytic enzymes in the infection of gerbera flowers by Botrytis cinerea. PhD. Thesis, University of Utrecht, The Netherlands.

Sambrook, J., Fritsch, E. F., and Maniatis, T. 1989. Molecular Cloning: A Laboratory Manual. 2nd ed. Cold Spring Harbor Laboratory Press, Cold Spring Harbor, NY

Shirane, N., Masuko, M., and Hayashi, Y. 1988. Nuclear behavior and division in germinating conidia of Botrytis cinerea. Phytopathology 78:1627-1630.

Shishiyama, J., Araki, F., and Akai, S. 1970. Studies on cutin-esterase II. Characteristics of cutin-esterase from Botrytis cinerea and its activity on tomato-cutin. Plant Cell Physiol. 11:937-945.

Stahl, D. J., Theuerkauf, A., Heitefuss, R., and Schäfer, W. 1994 Cutinase of Nectria haematococca (Fusarium solani f. sp. pisi) is not required for fungal virulence or organ specificity on pea. Mol. PlantMicrobe Interact. 7:713-725.
Sweigard, J. A., Chumley, F. G., and Valent, B. 1992. Disruption of a Magnaporthe grisea cutinase gene. Mol. Gen. Genet. 232:183-190.

Van den Ackerveken, G. F. J. M., Dunn, R. M., Cozijnsen, T. J., Vossen, P., Van den Broek, H. W. J., and De Wit P. J. G. M. 1994. Nitrogen limitation induces expression of the avirulence gene $a v r 9$ in the tomato pathogen Cladosporium fulvum. Mol. Gen. Genet. 243:277-285.

Van der Vlugt-Bergmans, C. J. B., Brandwagt, B. F., Van 't Klooster, J. W., Wagemakers, C. A. M., and Van Kan, J. A. L. 1993. Genetic variation and segregation of DNA polymorphisms in Botrytis cinerea. Mycol. Res. 97:1193-1200.

Van der Vlugt-Bergmans, C. J. B., Wagemakers, C. A. M., and Van Kan, J. A. L. 1997. Cloning and expression of the cutinase A gene of Botrytis cinerea. Mol. Plant-Microbe Interact. 10:21-29.

Verhoeff, K. 1980. Infection and host-pathogen interactions. Pages 153 180 in: The Biology of Botrytis. J. R. Coley-Smith, K. Verhoeff, W. R. Jarvis, eds. Academic Press, New York.

Wubben, J. P., Joosten, M. H. A. J., and De Wit, P. J. G. M. 1994. Expression and localization of two in planta induced extracellular proteins of the fungal tomato pathogen Cladosporium fulvum. Mol. Plant-Microbe Interact. 7:516-524.

Yao, C., and Köller, W. 1995. Diversity of cutinases from plant pathogenic fungi: Different cutinases are expressed during saprophytic and pathogenic stages of Alternaria brassicicola. Mol. Plant-Microbe Interact. 8:122-130. 